\title{
NON-DESTRUCTIVE METHODS INTO HEALTH MONITORING OF TALL TYPE BUILDINGS OPERATING INTO HAZARDOUS ENVIRONMENT
}

\author{
Khikmat Saidov, Janusz Szpytko \\ AGH University of Science and Technology \\ Faculty of Mechanical Engineering and Robotics \\ A. Mickiewicza Av.30,30-059 Krakow, Poland \\ e-mail:ksaidov@student.agh.edu.pl,szpytko@agh.edu.pl
}

\begin{abstract}
The paper is focusing on selected problems of health monitoring of tall type buildings. The actual problem in structural health monitoring (SHM) is to find the damages and its location by performing some statistical pattern recognition based on the measured data termed as feature extraction. The damage caused by environmental loads should be repaired; otherwise, it will expand with time and might lead to complete system failure. Dynamic parameters, such as velocity, acceleration and displacement, play a significant role in determining the structure dynamics. This paper is focusing on smart wireless sensors application into health monitoring systems of tall type buildings operating into hazardous environment and selected methods for data analysis obtained from the monitoring system. Smart sensors can locally process measured data and transmit only the significant information via wireless communication. As a network, wireless sensors extend the capability.

Significant future developments of smart wireless sensors technology in tall type buildings in hazardous environment come by way of multi-disciplinary research efforts encompassing fields such as data analysing, signal processing, structural dynamics, motion and environmental sensing hardware, computational hardware, smart materials, data telemetry and statistical pattern recognition, as well as other fields yet to be defined.

Specification issues that require automation of the decision making process in the evaluation of the technical state of the tall type structure have been also discussed.
\end{abstract}

Keywords: structural health monitoring, smart wireless sensors, data analysis methods

\section{Introduction}

The automated Structure Health Monitoring system (SHM) is a subject of rapidly developing, especially in health monitoring of tall type buildings operating into hazardous conditions. The today urgent request of the SHM system is to automate monitoring and decision-supporting processes based on data collecting from structure of buildings (such the stress-strain, temperature and displacement of constructions results acting various types' loads, and others) and processing in real-time.

The structural health monitoring systems of tall type buildings help to keep required safety and to optimize the maintenance and operational activities of complex constructions. Information resulting from monitoring programs permits owners to refine the maintenance, the operation, the repair and the replacement of structures, based on objective and reliable data. Detection of ongoing damage can be used to identify deviations in design performance values. Monitoring data can be combined into structural management systems and increase the quality and cost effectiveness of decisions by providing unbiased and reliable information.

Two types of approaches are known: destructive and non-destructive methods, Fig. 1. Using both destructive and non-destructive methods, we have to follow several steps to make decision related to the actual technical state condition of particular tall type building. The solutions provide reliable information on the factual condition of a structure observes its evolution and reveals the appearance of new degradations. Concrete will creep and crack and steel will oxidize and may 
crack because of fatigue loading. The material's degradation is caused by mechanical factors (i.e. loading higher than theoretically assumed) and physical-chemical factors (i.e. corrosion of steel, penetration of salts and freezing of concrete, chlorides in concrete, etc.). A real-time picture of a building's current state and evolution can be achieved by perennially installing sensors and continuously measuring relevant parameters.

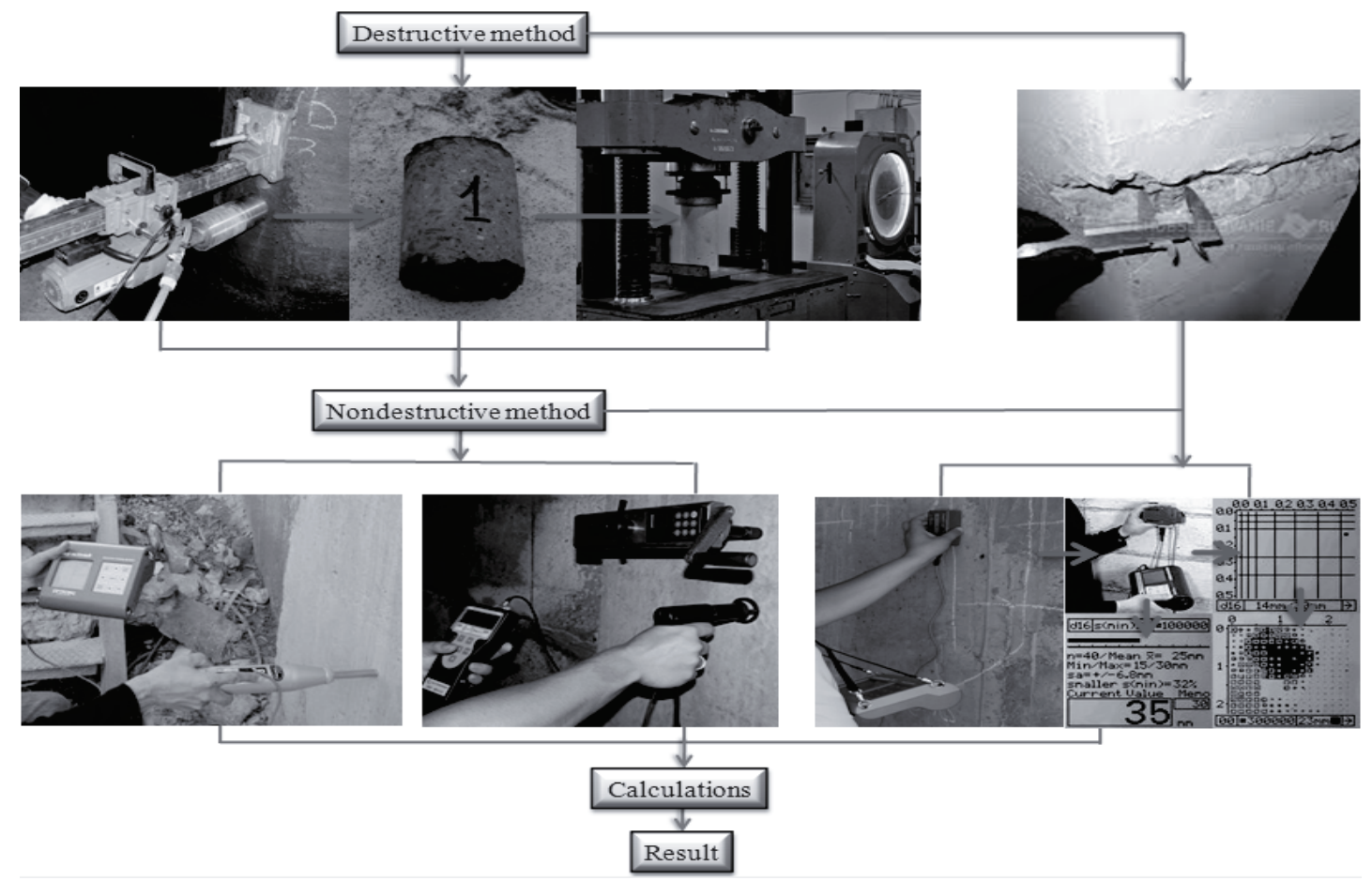

Fig. 1. Development of monitoring structures of buildings from destructive methods to non-destructive methods

The today importance is to develop fully automated systems supporting on-line type structure health decision-making system. The solution could be automated wireless monitoring system of the building. A monitoring system is expected installation on construction various types of sensors in order to determine the physical operation parameters (e.g. humidity and temperature, others) and acting forces (static and dynamic load types), which are results with strength and deformability.

Wireless monitoring systems of tall type structures in terms of the economy gives the possibility to reduce the cost of installing the wiring in the building, installation, commissioning works and others. Some of the reasons, which affect to increase, spend of the money on wired monitoring systems (shown in practices results the installation of monitoring systems at public facilities, such as bridges, towers, dams and others), is considerable negative impact of the environment. Resulting in we have to use moisture resistant cables and other protective measures, causing the cost of wired monitoring system increases dramatically.

This paper is focusing on smart wireless sensors application into health monitoring systems of tall type buildings operating into hazardous environment and selected methods for data analysis obtained from the monitoring system.

\section{Smart wireless sensors structure health monitoring}

Smart wireless sensors, with their on-board computational and communication capabilities, offer new opportunities for structure health monitoring (SHM) to automate monitoring acting loads in real time to the structure of tall type buildings. Without the need for power or communication cables, installation cost can be brought down drastically. History development of smart sensors has been presented on Fig. 2. 


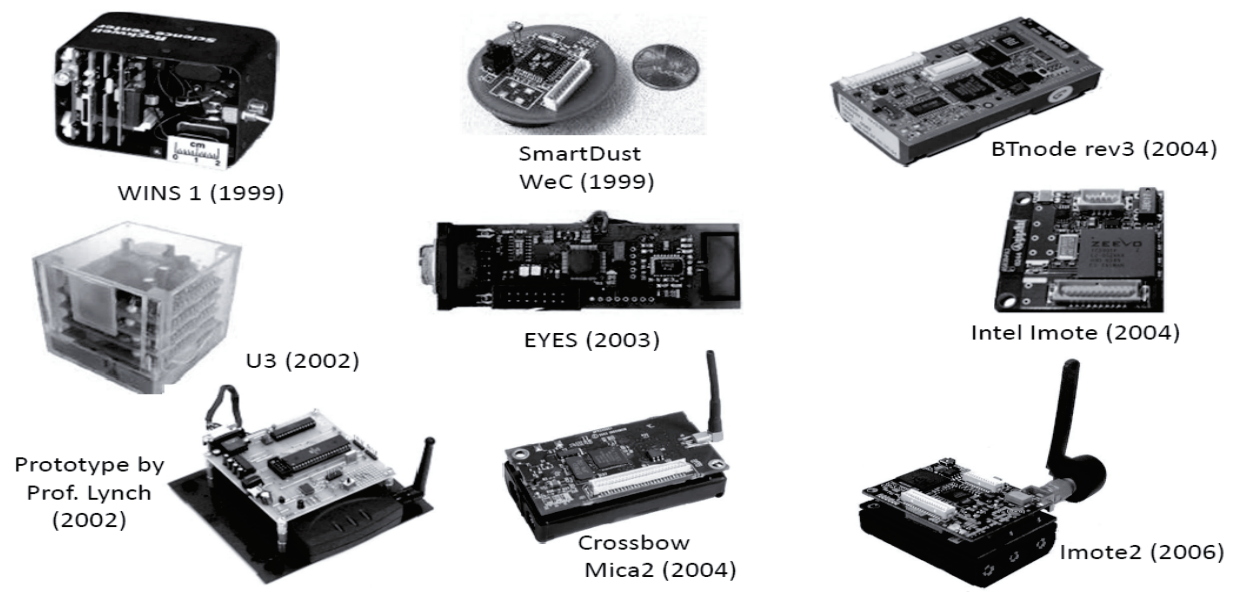

Fig. 2. History development of smart sensors [11]

Smart wireless sensors are able to help to make monitoring of structures with a dense array of sensors economically practical. Densely installed smart wireless sensors are expected to be abundant information sources for SHM [2], Fig. 3.

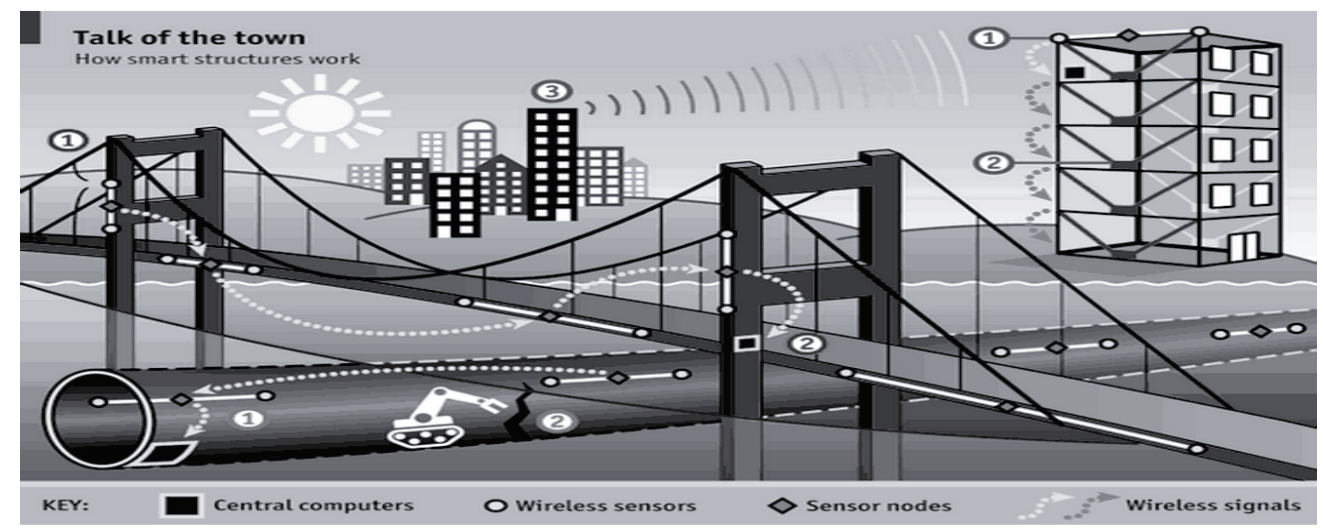

Fig. 3. Spheres, that use smart wireless sensors [13]: 1 - sensors to monitor the structure movement in response to strong winds or earthquake tremors; 2 - shock absorbers (hydraulic dampers) to stiffen or relax the construction with weights (mass dampers) to reduce oscillations in strong winds, or minimize damage in the event of an earthquake; 3 - buildings that detect an earthquake tremor could even warn other buildings nearby of the approach of a shockwave, so they could sound an alarm and prepare themselves accordingly

Wireless sensors mounded on the bridge monitor vibrations, displacement and temperature. This information then hops across the network of sensor nodes to a central computer for analysis. If a problem is detected, such as a loose bolt or cable, or the crack, a warning can be sent to operator by SMS. Wireless sensors mounted on the walls of a tunnel monitor displacement, temperature and humidity. This information then hopes across the network of sensors nodes to a central computer for analysis. If a problem with the tunnel lining is detected, appropriate maintenance can be carried out.

Efforts toward realization of SHM systems applying smart sensors, however, have not resulted in full-fledged applications. All efforts to date have encountered difficulties originating from limited resources on smart sensors (e.g., small memory size, small communication throughput, limited speed of the CPU, etc.). To realize an SHM system employing smart sensors, this system needs to be designed considering both the characteristics of the smart sensor and the structures to be monitored [2].

The physical state of a structural system, for instance, applied load, vibration level, and existence of structural damage, is among the factors that identify serviceability. Sensing physical quantities in detail proposes the potential to better evaluate structural conditions. For riverbank 
protection, for example, water level can be monitored and the associated load evaluated. Precipitation rate and groundwater level are significant indicators to forecast slope failure. Temperature and strain measurements can be utilized to monitor concrete gravity or arch dams. Users, engineers, and owners can make better decisions depends on the measured data [2].

Because buildings, towers and bridges are typically complex and large, data from just a few sensors is incorrect to accurately assess the structural condition. The dynamic behaviour of these structures is complex in both time scale and spatial. Furthermore, deterioration/damage is intrinsically a local phenomenon. Consequently, to comprehend such dynamic behaviour, the motion of structures needs to be monitored by densely located sensors at a sampling frequency sufficiently high to capture salient dynamic characteristics [2].

When massive sensors have been implemented, wireless communication seems to be attractive. The high cost connected with the installation of wired sensors [1, 4], can be vastly reduced by employing wireless sensors. Wireless sensors frequently convert analog signals to digital signals prior to radio frequency (RF) transmission, while many wired systems collect analog signals at one or several base stations where the signals conversion takes place. The digital conversion on the wireless sensor node excludes possible signal degradation during analog signal communication through long cables. Thus, wireless sensor systems are promising as data acquisition systems with a wide number of sensors installed on sizable structures.

Being smart, or having data processing capability in the sensors, is an important feature that further enhances the potential of wireless sensors. Smart sensors can locally process measured data and transmit only the significant information via wireless communication. As a network, wireless sensors extend the capability. For example, sensors that are malfunctioning in the network can be detected, and other sensors can reconstruct sensor topology without this dead node. As another example, location mapping can be implemented automatically by a localization service [3], which helps civil engineers identify and confirm the location of wide numbers of sensors on complex structures.

\section{Existing problems of smart wireless sensors}

Smart wireless sensors, however, have bounded resources, disallowing direct application of traditional structural monitoring strategies. For instance, the communication speed is very slow for centrally collect all of the measured information. Clocks on sensor nodes are not always synchronized. Some communication packets may be lost. Storage and memory space is limited. Processor speed is slower than that of a PC (Personal Computer). Smart sensors do not necessarily offer a real-time system; programmers may not be able to assign appropriate priority to given tasks. Additionally, battery power imposes limitations on many aspects of smart sensors. Any task consuming huge amounts of power becomes impractical on a battery-operated smart sensor node. Smart sensor systems need to overcome these limitations using deliberate system design, as seen in some of the time synchronization and authentic communication research efforts [8].

So far, many smart sensor prototypes have been developed and several attempts to use smart sensors for SHM. Most attempts at SHM with smart sensors only replace the wired link with wireless communication and apply traditional damage detection algorithms at the base station. An SHM system with such algorithms assuming central data collection does not scale to a wide number of smart sensors. Some researchers have also proposed approaches where simple data processing is performed on each smart sensor node without interaction with other nodes; these attempts do not employ spatial data, and, therefore, have room for improvement in terms of damage detection capability. Information from a dense array of smart sensors has to be processed in a coordinated manner, rather than independently. SHM algorithms for distributed and coordinated data processing making use of the smart sensor's distributed computing and sensing resources have only recently appeared. 


\section{Selected methods for data analysis}

While designing a structural health monitoring (SHM) system, one should always focus on the certain requirements of the structure under test. The first step in the design process consists in identifying the probable degradation mechanism and the associated risks factors, in collaboration with the structure's designer and owner. Consequently, the expected responses to these degradations are established and an appropriate structural health monitoring systems is designed to reveal such conditions. Only at this stage, the appropriate sensors should be selected.

Once the sensors are verified and installed, data collection can start. If these logical steps are followed and the monitoring data is adequately acquired and managed, data analysis and interpretation will be immensely simplified. In addition, if one designs an SHM system starting from a specific sensor system, it frequently ends up with a great quantity of data, but no plans on how to analyse it. When selecting the best suitable sensors for the specific risks associated with a given structure, it is often important to integrate different measurement technologies.

The use of relational database structures can immensely oversimplify the handling of this large and heterogeneous data-flow. With an adequate data structure, the measurement data and other related information on the monitoring network, the structure and its environment can be organized in a single repository that will follow the structure's life in the years and near future.

The aim of structural health monitoring for civil structural engineering is not only detection of sudden or progressive damages but also monitoring their performance under operational conditions or under some particular environmental issues such as earthquakes [7].

One of the essential aspects for the application of damage detection techniques as a part of monitoring practices is an automated identification and tracking procedure, because traditional modal identification requires extensive interaction from an experienced user [9].

Currently, there are few advancements in this field, with the development of methods based on control theory and methods based on conventional signal processing. As regards the first class of methods, during modal analysis the model order is usually over-specified to get all physical modes present in the frequency range of interest. Nevertheless, physical and mathematical modes have to be refined.

This practice requires large interaction with an expert user. In addition, most of the classical model order selection tools used in time domain and in frequency domain identification only allow to verify if the model order used is appropriate but do not separate physical from mathematical modes [10]. Thus, the stabilization diagram is yet significant tool in modal analysis to separate physical from mathematical modes. Selection of physical poles is not however a trivial tasks, since it might be difficult and time-consuming depending on the quality of data obtained, the performance of the estimator and the experience of the user. Extensive interaction between tools and user is generally inappropriate for monitoring purposes [6], Fig. 4.

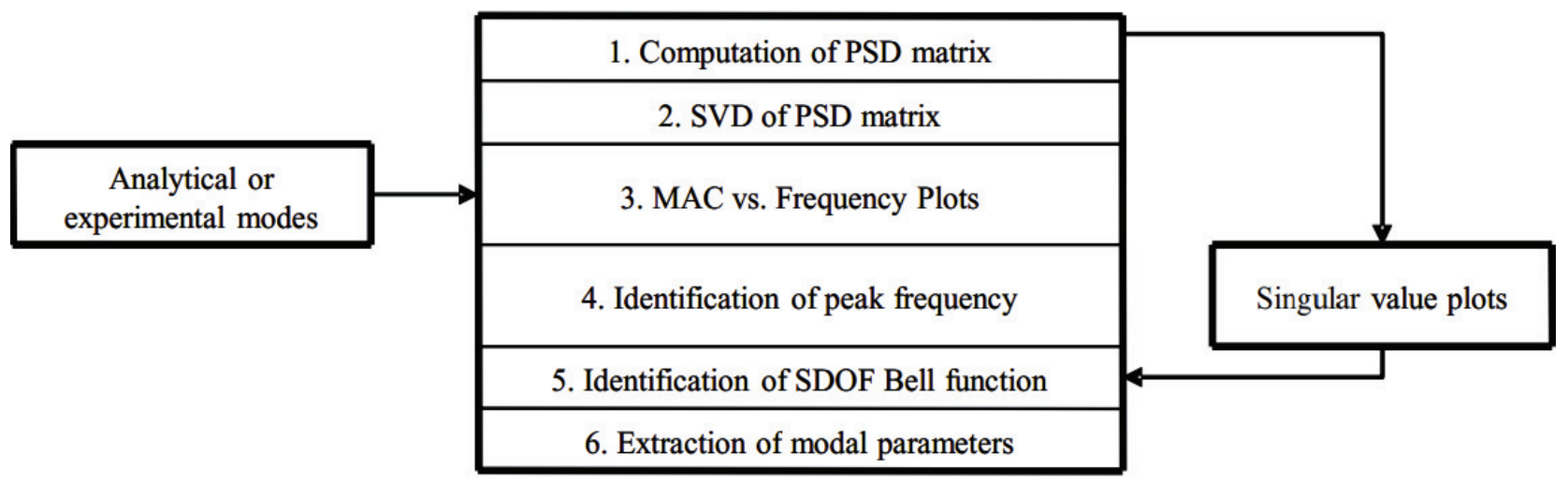

Fig. 4. The algorithm for automated modal identification [6, 9] 
The algorithm for automated modal identification presented on Fig. 2 is based on Enhanced Frequency Domain Decomposition (EFDD for the modal identification in operational conditions. Identification of the auto power spectral density function of the corresponding Single Degree Of Freedom (SDOF) system by comparing the mode shape estimate with the singular vectors at the frequency lines around the peak after defining a Modal Assurance Criterion (MAC) rejection level. The Inverse Fourier Transform (IFT) of the identified spectrum (SDOF Bell function) for the corresponding SDOF system allows the computation of the damping ratio by the logarithmic decrement technique. The described procedure can be automated if the mode shapes (experimental or numerical) of the monitored structure are known according to the procedure shown in Fig. 2.

As in the time domain, the output of a linear dynamic system can be expressed in terms of its mode shapes and generalized coordinates. Performing the SVD (singular value decomposition) of the PSD (positive definite) matrix it is decomposed into a set of auto-spectral density functions, each corresponding to a SDOF system. Since the peaks of the SV (singular values of frequency) plot indicate a mode (when spurious harmonic modes do not exist) and the singular vector associated to the peak frequency is an approximation of the true mode shape. In fact, the plot of MAC vs. frequency will illustrate an absolute maximum at the frequency of the mode itself, where the singular vector obtained from the SVD of the PSD matrix approximates the effective mode shape of the structure. After the identification of the peak frequency, the mode characterization is performed according to the standard EFDD procedure $[6,9]$.

According to paper [49], an interesting proposal for automated modal identification based on Least Square Complex Frequency (LSCF) method has been presented. This method moves from the identification of a model with sufficiently high order using a frequency - domain Maximum Likelihood estimator. A first validation of the poles is performed on the base of both stochastic and deterministic criteria, which allow the identification and removal of a first group of non physical poles.

Consequently, as regards the methods based on conventional signal analysis in paper [5] authors have proposed the so-called Time Domain Filtering method, which is based on the application of a band-pass filter to the system response with the aim of isolating the single modes in the spectrum. However, the frequency limits of the filter are user-specified based on the Power Spectral Density (PSD) plots of the response signals and, if excitation is obscure, it is sometimes complicated to identify the regions where certain modes may be located according only to power spectrum plots.

A significant challenge in developing an SHM strategy for civil infrastructure is that except for certain types of public and private housing, every structure is unique. This means that there is no baseline derived from type testing or the expensive qualification procedures applicable for aerospace structures. Thus, a unique feature of SHM for civil infrastructure is that a major part of the system has to be geared towards a long-term evaluation of what is normal structural performance or health, the two terms being synonymous.

Specification issues that require automation of the decision making process in the evaluation of the technical state of the tall type structure cover:

1. Damage detection on the basis of influence coefficients. This method uses a time-domain identification procedure to detect structural changes on the basis of noise-polluted measurements. Application of the identification procedure under discussion yields the optimum value of the elements of equivalent linear system matrices (influence coefficients). By performing the identification task before and after potential structural changes (damage) in the physical system have occurred, quantifiable changes in the identified mathematical model may be detected by analysing the probability density functions of the identified system matrices.

2. Damage detection using neural networks. Among the structure-unknown (model-free) identification approaches that have been receiving growing attention recently are neural networks. Not only do neural networks not require information concerning the phenomenological nature of the system being investigated, they also have fault tolerance, 
which makes them a robust means for representing model-unknown systems encountered in the real world.

3. Reliability and risk analysis. The model updating methodology based on a nonlinear physicsbased model of the monitored structure will be used not only as a tool for tracking the health of the structure, but also as a basis to assess the reliability of the structure in performing as expected under uncertain current and future loads. Reliability of the structure against various potential limit-states can be evaluated using a probabilistic mechanics-based model of the structure and a probabilistic representation of current and future load effects and deterioration effects. The combination of probabilistic non-destructive structural health monitoring techniques and computational methods of reliability analysis provides a powerful tool to continuously monitor competency of the structure under consideration.

4. Probabilistic modelling and computational decision theory. Probabilistic networks (or Bayesian probabilistic networks) provide a comprehensive framework for modelling and analysing uncertainties. Although there have been a number of developments in this field, there are still numerous challenges in extending the theory and tools to address a larger range of applications, including the incorporation of background knowledge into the model-building process, providing large-scale database support for probabilistic modelling and decision support, and relating probabilistic modelling to other mathematical and statistical methods.

\section{Conclusions}

An essential advantage of wireless sensors over other sensors, such as fiber optic sensors, is the collocation of computational power with the sensing transducer. In fact, this feature transforms the wireless monitoring system into a genuine structure health monitoring system where damage detection is completely automated. Nowadays, many engineering algorithms, including wavelet transforms, Fourier transforms and system identification models, have been embedded. Nevertheless, wireless sensors should be considered as a decentralized architecture offering parallel processing of measurement data. More effective research is needed to arrive at truly distributed data interrogation schemes designed explicitly for the decentralization and parallelism offered by wireless sensors.

Finally, an important challenge for SHM is to develop the capability to define the required sensing system properties before field deployment and, if possible, to demonstrate that the sensor system itself will not be damaged when deployed in the field. If the possibility of sensor damage exists, it will be necessary to monitor the sensors themselves. This monitoring can be accomplished either by developing appropriate self-validating sensors or by using the sensors to report on each other's condition. Sensor networks should also be fail-safe. If a sensor fails, the damage identification algorithms must be able to adapt to the new network. This adaptive capability implies that a certain amount of redundancy must be built into the sensor network.

Significant future developments of smart wireless sensors technology in tall type buildings in hazardous environment come by way of multi-disciplinary research efforts encompassing fields such as data analysing, signal processing, structural dynamics, motion and environmental sensing hardware, computational hardware, smart materials, data telemetry and statistical pattern recognition, as well as other fields yet to be defined. These topics are the focus of important discipline-specific research efforts, and it is the authors' speculation that to date not all technologies from these fields that are relevant to the SHM problem have been explored by the research community.

Furthermore, there are few efforts that try to advance and combine these technologies with the specific focus of developing SHM solutions. Without such a focus in mind, these technologies may not evolve in a manner that is not necessarily optimal for solving the SHM problem. Eventually, the problem of global SHM of civil and industrial buildings and facilities is significantly complex and diverse that it will not be solved in the immediate future. Like so many 
other technology fields, advancements in SHM will most likely come in small increments requiring diligent, focused and coordinated research efforts over long periods of time.

\section{References}

[1] Celebi, M., Seismic instrumentation of buildings (with emphasis on federal buildings) Special GSA/USGS Project, an administrative report, United States Geological Survey, Menlo Park, CA, 2002.

[2] Cho, S., Yun, C., Lynch, J. P., Zimmerman, A. T., B. F. S. Jr, and T. Nagayama, Smart Wireless Sensor Technology for Structural Health Monitoring of Civil Structures, Vol. 8, pp. 267-275, 2008.

[3] Doherty, L., Pister, K. S. J. \& Ghaoui. L. E., Convex position estimation in wireless sensor networks, Proceedings of the IEEE Infocom, AK., 1655-1663, 2001.

[4] Farrar, C. R., Historical overview of structural health monitoring. Lecture Notes on Structural Health Monitoring using Statistical Pattern Recognition, Los Alamos Dynamics, NM, 2001.

[5] Guan, H., Karbhari, V. M., Sikorski, C. S., Time-domain output only modal parameter extraction and its application, Proceedings of the 1st BIOMASS Conference, Copenhagen, Denmark 2005.

[6] Lanslots, J., Rodiers, B., Peeters, B., Automated Pole-Selection: Proof-of-Concept and Validation, Proceedings, International Conference on Noise and Vibration Engineering (ISAM) Leuven, Belgium 2004.

[7] Mufti, A., Guidelines for structural health monitoring, University of Manitoba, ISIS, Canada 2001.

[8] Maroti, M. Kusy, B., Simon, G., \& Ledeczi, A., The flooding time synchronization protocol, Proceedings of 2nd International Conference On Embedded Networked Sensor Systems, Baltimore, MD, 39-49, 2004.

[9] Ou, J., The state-of-the-art and application of intelligent health monitoring systems for civil infrastructures in mainland of China, In: Progress in structural engineering, mechanics and computation, Zingoni, A., (ed.), pp. 599-608, Balkema/ Taylor and Francis, London 2004.

[10] Rainieri, C., Fabbrocino, G., Cosenza E., Automated operational modal analysis as structural health monitoring tool: theoretical and applicative aspects, Key Engineering Materials, Vol. 347, pp. 479-484, 2007.

[11] Soderstrom, T., Test of pole-zero cancellation in estimated models, Automatica, Vol. 11 (5), pp. 537-541, 1975.

[12] Spencer, B. F., Structural Health Monitoring of Civil Infrastructure: From Research to Engineering Practice, Nathan, M., Anne M., Newmark Endowed Chair in Civil Engineering, Director, Newmark Structural Engineering Laboratory, Director, MUST-SIM: NEES@Illinois, Director, Smart Structures Technology Laboratory (SSTL), 2013.

[13] http://www.rfwirelesssensors.com/. 